INTERVIEW

For reprint orders, please contact: reprints@futuremedicine.com

\title{
Personalized medicine: the future of breast cancer management
}

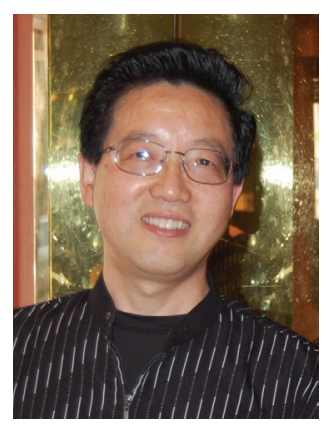

Zhixiang Wang* speaks to Gemma Westcott, Commissioning Editor: After obtaining his PhD, Zhiziang Wang did his postdoctoral training at the University of Toronto (ON, Canada) supported by various scholarships including the Canadian Institutes of Health Research's Centennial Fellowship, Charles H. Best Postdoctoral Fellowship and The Natural Sciences and Engineering Research Council of Canada Postdoctoral Fellowship. He started his first independent research position as a Career Scientist at Northeastern Ontario Regional Cancer Center (NEORCC) (ON, Canada) in 1996 and as an Assistant Professor at University of Ottawa (ON, CA) in 1997. He moved to the Department of Cell Biology, University of Alberta (AB, Canada) as an Assistant Professor. He is now a Full Professor at the Department of Medical Genetics, University of Alberta. His research has focused on ErbB receptor-mediated cell signaling, receptor endocytosis and human cancer. ErbB receptors including EGFR/ErbB1, ErbB2, ErbB3 and ErbB4 lie at the head of a complex signal transduction cascade that modulates cell proliferation, survival, adhesion, migration and differentiation. While ErbB receptor signaling is essential for many normal cell functions, the aberrant activity of ErbB receptors has been shown to play a key role in the development of many cancers. ErbB receptors are overexpressed in many cancers especially in breast cancer, ovarian cancer, small cell lung cancer and skin cancer. ErbB receptor overexpression correlates to poor prognosis, drug resistance, cancer metastasis and lower survival rate. All these make ErbB receptor the top choice as a target for developing cancer therapies. To date, monoclonal antibodies (mAbs) and synthetic inhibitors of tyrosine kinase have taken central stage. The central theme of his research is to understand how the activation of EGFR regulate cell signaling, how the signaling is terminated through EGFR endocytosis, trafficking and degradation, how the breakdown of this regulation contributes to cancer development and how an intervention can be provided.

Q Do you mind telling our readers about your career to date?

I did my undergraduate study and got my BSc degree at Beijing University (Peking University) in 1982. I also obtained an MSc degree in Zoology at Chinese Academy of Science. I went to Simon Fraser University (BC, Canada) in 1989 to pursue my $\mathrm{PhD}$ in the Department of
Biological Science. After graduation with a PhD degree and a Dean's medal in 1993, I did 3 years postdoctoral training at the University of Toronto. I started my independent research at NEORCC as a Career Scientist for 2 years. I have been a professor at University of Alberta since 1999. As an independent researcher, my research has been focusing on ErbB receptor-mediated

*Department of Medical Genetics \& Signal Transduction Research Group, Faculty of Medicine \& Dentistry, University of Alberta, Edmonton, Alberta T6G 2H7, Canada; zhixiang.wang@ualberta.ca

\section{KEYWORDS}

- breast cancer $\bullet$ drug resistance

- ErbB receptor overexpression

- personalized medicine 
cell signaling and its implication in breast cancer.

Q What was it that sparked your research interest in the cell signaling underpinning breast cancer?

I began to study EGFR (ErbB1)-mediated cell signaling and EGFR endocytosis during my postdoctoral fellow year in Toronto. As you know, EGFR signaling is critical for many normal cell functions and has broad implications in many cancers including small cell lung cancer, skin cancer, breast cancer and ovarian cancer etc. I began to show interest in breast cancer when I moved to NEORCC at the end of 1996. When you are doing basic biomedical research in a cancer center, you feel obligated to link your research to cancer and make a contribution to cancer management. You also have a lot of opportunity to interact with oncologists. Breast cancer is the most common cancer for woman. A significant portion of breast cancer has shown to overexpress ErbB receptors including EGFR, ErbB2 and ErbB3. At NEORCC, I began to link my research to breast cancer and published a couple of papers related to breast cancer.

\section{Q Can you pick out a favorite moment in your career?}

My favorite career period was during 1994 to 1996, when I switched my research from insect neurobiology and biochemistry to biomedical research. I had been very productive in my old field with a total publication of 18 papers in 5 years. However the resources were limited and I found the job perspective to be quite low within insect neurobiology and biochemistry. With the encouragement of my wife (she switched to biomedical field earlier), I decided to switch to the field of biomedical research. It turned out to be a very good decision. Within 2 years in the biomedical research field, I published two good papers including one in Science regarding the regulation of EGFR endocytosis by signaling protein Grb2. I was also awarded the Medical Research Council Centennial Fellowship (specific for potential independent researcher). With a promising bright future ahead, we also started our family with our first boy born in November 1995. During that period I worked very hard, but with a very high spirit. I also set out my future research and new career direction.
Q You spoke recently at the Controlling Cancer Summit 2015 (London, UK) about your work on chemo-resistance in breast cancer. What are the major obstacles in preventing this resistance currently?

In my view, three major obstacles. First, lack of knowledge regarding the molecular mechanisms of resistance. We have to acknowledge the progress in understanding resistance, especially the discovery of multidrug resistance. However, multidrug resistance proteins are certainly only the part of the story. It has long been known that specific cancer patients may develop specific resistance mechanisms toward specific chemotherapy. For example, our data suggest that expression levels of various $\beta$-tubulin isotypes may confer the resistance to taxanes in breast cancer. Recent research regarding cancer stem cells suggests that the existence of cancer stem cells may be the reason of drug resistance. Second, the lack of effective new therapeutic agents. While the new chemotherapeutic drugs are getting to the market faster than ever, it is still far from enough. With limited choice, the same drugs have to be repeatedly used, which increases the chance to develop drug resistance. Third, the heterogeneity of the cancer including breast cancer means the presence of various drug resistance mechanisms. It is very difficult - if not impossible - to find a way that can overcome all of the drug resistance.

\section{Q How does ErbB receptor overexpression contribute to this resistance and what research is currently being done?}

While it is well-established that overexpression of ErbB receptors confers resistance to various chemotherapies, the underlying mechanisms are not completely clear. In general, there are many ways that ErbB receptor overexpression contributes to drug resistance. First, the cells with overexpressed ErbB receptors generate strong surviving signals, which allow the cells to be more resistant to drug-induced cell death. Second, the breast cancer cells overexpressing ErbB receptors have a much higher proliferation rate due to ErbB receptor-mediated cell signaling, which may dwarf drug-induced cell deaths. Finally, breast cancer cells overexpressing more than one ErbB receptors could be resistant to any therapy targeting one ErbB receptor due to the crosstalk between different ErbB receptors and the redundant signaling pathways mediated by various ErbB receptors. 
Q Would you say personalized medicine based on individual patient molecular profiles is the future of breast cancer management? I certainly believe that personalized medicine based on the molecular profiles of individual patients is the future of breast cancer management. In fact, we have made significant progress in this direction. The classification of breast cancer into five intrinsic subtypes based on the molecular profiles of the breast cancer cells, over a decade ago, is the first and a very important step toward personalized medicine for breast cancer. Based on this classification, different subtypes of breast cancer are treated differently. For example, trastuzumab and pertuzumab are employed to treat ErbB2-positive breast cancer. Tamoxifen is used to treat estrogen receptor (ER)-positive breast cancer. Further development of personalized medicine is certainly critical to improve our ability to manage breast cancer.

Q How can we move forward in personalized treatment strategies for breast cancer?

In my view, further subgrouping of breast cancer based on identified biomarkers could gradually move us forward to real personalized treatment of individual breast cancer patient. For example, we can subdivide ErbB2-positive breast cancer into ER-positive and ER-negative subgroups. We can further subgroup either ER-positive or ER-negative subgroup based on the expression profiles of ErbB receptors including EGFR and ErbB3.

However, to be able to accurately further subdivide the breast cancer subtypes, identification and validation of significant numbers of biomarkers are required. This cannot be achieved overnight and requires enormous funding and long-term efforts.

\section{Q Where is your research heading?}

The major direction of my research is to explore the possibility of subdividing ErbB2-positive breast cancer based on the expression level of various ErbB receptors, and to develop optimum treatment strategies for each subtype using the existing therapeutic agents. To achieve this, we are now focusing our research on the understanding of the molecular mechanisms of trastuzumab and pertuzumab and on the application of this knowledge to design better therapies. Another project is to understand the regulation of EGFR signaling during cell cycle, especially in mitosis. The goal is to explore the possibility of targeting mitosis-specific EGFR signaling to provide novel and better therapies for EGFRpositive triple negative breast cancer. As you know, nearly $50 \%$ triple negative breast cancer is EGFR positive.

Q Finally, where would you like to see the field of breast cancer research in 10 years' time?

I would like to see significant numbers of new drugs passing through clinical trials and coming to the market, with also many more biomarkers being identified and validated. A much better understanding is needed of the molecular mechanisms underlying breast cancer development, the function of available therapeutic drugs and of drug resistance. On this basis, I would like to see a big improvement of personalized medicine for breast cancer management. For example, each of the current five subtypes of breast cancer may be further divided into several or more subtypes based on existing and newly identified biomarkers. Treatment should be readily available for each of these subtypes of breast cancer. If all this happens, we will significantly lower the mortality of breast cancer.

\section{Financial \& competing interests disclosure}

The author has no relevant affliations or financial involvement with any organization or entity with a financial interest in or financial conflict with the subject matter or materials discussed in the manuscript. This includes employment, consultancies, honoraria, stock ownership or options, expert testimony, grants or patents received or pending, or royalties.

No writing assistance was utilized in the production of this manuscript. 